\title{
Arbitrary Steady-State Solutions with the K-Epsilon Model
}

\author{
C. L. Rumsey \\ NASA Langley Research Center, Hampton, Virginia, 23681-2199, USA \\ B. A. Pettersson Reif \\ Norwegian Defence Research Establishment, P.O. Box 25, NO-2027, Kjeller, Norway \\ T. B. Gatski \\ NASA Langley Research Center, Hampton, Virginia, 23681-2199, USA \\ Submission to AIAA Journal \\ Widely-used forms of the $K$ - $\varepsilon$ turbulence model are shown to yield arbitrary steady- \\ state converged solutions that are highly dependent on numerical considerations such \\ as initial conditions and solution procedure. These solutions contain pseudo-laminar \\ regions of varying size. By applying a nullcline analysis to the equation set, it is pos- \\ sible to clearly demonstrate the reasons for the anomalous behavior. In summary, the \\ degenerate solution acts as a stable fixed point under certain conditions, causing the \\ numerical method to converge there. The analysis also suggests a methodology for \\ preventing the anomalous behavior in steady-state computations.
}

\section{Introduction}

The prediction of turbulent flow fields for engineering purposes continues to be dominated by employing the Reynolds-averaged Navier Stokes (RANS) approach. Although there are several classes of closure methodologies available, the class of two-equation linear eddy viscosity models may be the most popular. Included in this class is the $K-\varepsilon$ model, which has many variants. Such models are most effectively utilized when the focus is on mean field dynamics 
rather than detailed behavior of the statistical moments. The two-equation formulation yields an eddy viscosity that directly influences the mean flow behavior. The $K$ equation can be directly derived from the full Reynolds stress transport equation (by taking the trace). Closure of this $K$ equation then requires a constitutive equation for the Reynolds stress tensor as well as the transport and pressure-diffusion terms. For the Reynolds stress tensor, a linear relation with the mean strain rate tensor is assumed with the proportionality coefficent being the turbulent eddy viscosity. For the transport and pressure-diffusion terms gradient transport models are generally assumed. The $\varepsilon$ equation is based on transport processes in the dissipative range, but it is often viewed as being an empirical model. ${ }^{1,2}$

Naturally, implicit in the use of all RANS models is the assumption that for a given set of initial and boundary conditions, a unique solution will be obtained. However, it will be shown here that for certain formulations of the $K-\varepsilon$ model, portions of the flow field can converge to a degenerate solution that is "pseudo-laminar" in nature. The terminology "pseudo-laminar" alludes to the fact that the model does not predict the correct laminar limit in terms of the turbulent to mean flow time scale $(S K / \varepsilon)$, but the resulting eddy viscosity is still near zero so the mean flow behaves as a laminar flow. Unfortunatley, this disturbing behavior is further exascerbated by the fact that the location and spatial extent of these pseudo-laminar regions in the converged solutions can depend on initial conditions and method of solution! This paper both demonstrates the problem and performs an analysis that explains why the system of equations behaves in this manner. It should be noted that this anomalous behavior occurs only in developing flows, i.e., boundary layers. Flow configurations that utilize streamwise periodic boundary conditions (such as fully developed channel flow) do not in general encounter this problem.

Such issues have not been addressed previously, and the purpose of this analysis is to critically examine the characteristics of the $K-\varepsilon$ model from a dynamical systems standpoint. Mohammadi and Pironneau ${ }^{3}$ reported extensive mathematical analysis on the $K-\varepsilon$ model, but did not consider the anomalies described here. Dynamical system analyses have previously been utilized $^{4-6}$ in order to identify the fixed points of two-equation and second-moment closures in homogeneous shear flow, and to calibrate closure models for equilibrium flows. The issues addressed in the present study, however, require that the evolution toward the equilibrium state is understood, particularly the solution trajectories from a given initial condition, and not the fixed points per se. Although the theoretical approach is inherently based on the simplifying assumption of homogeneous turbulence, an attempt is also made to account for inhomogeneous effects so that the theoretical results can be more easily related to the full numerical solution of the model in realistic wall-bounded flows. 
As already alluded to, the dynamical systems analysis undertaken here is not only concerned with fixed point identification but also about dependent variable phase-plane trajectories and dependence on initial conditions. The analysis is shown to isolate deficiencies in some formulations of the $K-\varepsilon$ model that lead to initial condition and solution-method dependent converged solutions.

\section{Illustration of the Problem}

To illustrate the anomalies arising in numerical solutions of the $K-\varepsilon$ model, two cases are considered: (1) a flat plate boundary layer flow, and (2) flow over an airfoil. The computer

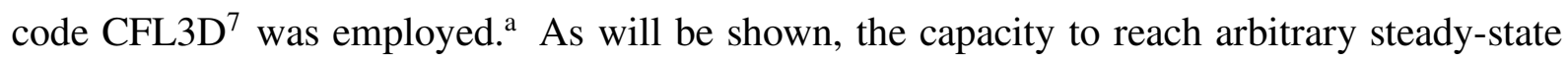
solutions is a property of the $K-\varepsilon$ equations themselves, so any numerical method will encounter the problem.

A basic form of the $K-\varepsilon$ model can be written as

$$
\begin{aligned}
\frac{D K}{D t} & =\mathcal{P}-\varepsilon+\frac{\partial}{\partial x_{j}}\left[\left(\nu+\frac{\nu_{t}}{\sigma_{K}}\right) \frac{\partial K}{\partial x_{j}}\right] \\
\frac{D \varepsilon}{D t} & =\frac{\varepsilon}{K}\left(C_{\varepsilon 1} \mathcal{P}-f_{2} C_{\varepsilon 2} \varepsilon\right)+\frac{\partial}{\partial x_{j}}\left[\left(\nu+\frac{\nu_{t}}{\sigma_{\varepsilon}}\right) \frac{\partial \varepsilon}{\partial x_{j}}\right],
\end{aligned}
$$

where $D / D t=\partial / \partial t+U_{j} \partial / \partial x_{j}, C_{\varepsilon 1}=1.44, C_{\varepsilon 2}=1.83, \sigma_{K}=1.0, \sigma_{\varepsilon}=\kappa^{2} /\left[\sqrt{C_{\mu}}\left(C_{\varepsilon 2}-\right.\right.$ $\left.C_{\varepsilon 1}\right)$ ], $\kappa=0.41$, and $C_{\mu}=0.09$. The set of values used here for the closure constants are representative of values typically chosen. However, as will be seen, the anomalous behavior exists regardless of their exact numerical values. The rate of turbulent energy production $\mathcal{P}$ is defined as $-\overline{u_{i} u_{j}} \partial U_{i} / \partial x_{j}$, and the components of the Reynolds stress tensor is modeled using the Boussinesq assumption,

$$
\overline{u_{i} u_{j}}=\frac{2}{3} K \delta_{i j}-\nu_{t}\left(\frac{\partial u_{i}}{\partial x_{j}}+\frac{\partial u_{j}}{\partial x_{i}}\right)
$$

with the eddy viscosity given by

$$
\nu_{t}=C_{\mu} \frac{K^{2}}{\varepsilon} .
$$

In this type of formulation, the function $f_{2}$ is introduced into Eq. (1b) so that as a solid boundary is approached the destruction-of-dissipation term does not become ill-behaved. ${ }^{9}$ It is

\footnotetext{
${ }^{\mathrm{a}}$ Another code, ISAAC, ${ }^{8}$ has also been used and yields similar results.
} 
also used to calibrate the model in the log-layer region. For example, two of the most widelyused forms for $f_{2}$ are:

$$
f_{2}=1-c_{1} \exp \left(-c_{2} R e_{T}^{2}\right)
$$

where $R e_{T}=K^{2} /(\nu \varepsilon)$, and

$$
f_{2}=1-c_{3} \exp \left(-c_{4} R e_{K}\right)
$$

where $R e_{K}=\sqrt{K} d / \nu$ and $d$ is the distance from the wall. The constants $c_{1}$ and $c_{3}$ are always positive and less than or equal to 1.0 , and $c_{2}$ and $c_{4}$ are positive constants. Note that $1-c_{1} \leq$ $f_{2} \leq 1$ for Eq. (4) and $1-c_{3} \leq f_{2} \leq 1$ for Eq. (5). For the present demonstrations, only Eq. (5) was used, with the commonly used values $c_{3}=1, c_{4}=2 / 25$, but Eq. (4) exhibits similar behavior.

In order to rule out the possibility that the observed arbitrary behavior occurred only for a particular set of boundary conditions, various freestream boundary conditions for $K$ and $\varepsilon$ were employed. The anomalies occurred regardless of these variations, as exemplified below. Because the solutions were highly dependent on numerical parameters, the grid size itself could also have an influence on the final solution. Reasonably fine grids were used for both cases, but a formal grid independence study was not conducted because it has no meaning when the equations themselves (and not the numerics) can yield arbitrary results.

\section{A. Flat Plate Boundary Layer}

In the flat plate boundary layer flow considered first, a freestream turbulent intensity of $0.2 \%$ $\left(=\sqrt{2 K_{\infty} / 3}\right)$ was imposed everywhere in the computational domain using a grid of $193 \times 65$ at a Reynolds number (based on plate length) of $R e=6 \times 10^{6}$. Thus, the initial condition $K_{0}$ on $K$ was everywhere the same as the boundary condition $\left(K_{0}^{\prime}=K_{\infty}^{\prime}=K_{\infty} / u_{\infty}^{2}=6.0 \times 10^{-6}\right)$. The dissipation rate boundary condition at inflow, which determines the freestream eddy viscosity and turbulence freestream decay rate, was set at $\varepsilon_{\infty} l / u_{\infty}^{3}=8.1 K_{\infty} / u_{\infty}^{2}$, or $\varepsilon_{\infty}^{\prime}=8.1 K_{\infty}^{\prime}$. This yielded a freestream eddy viscosity for this case of $\mu_{t} / \mu_{\infty}=0.40$ at the boundaries. A sample solution showing the final $u / U_{\infty}$-velocity contours is plotted in Fig. 1, for the case with initial condition $\varepsilon_{0}^{\prime}=8.1 K_{0}^{\prime}$. The solution exhibited a pseudo-laminar solution upstream of $x / l \approx 0.1$, then a turbulent solution downstream. Next, different cases were run with four different initial conditions $\varepsilon_{0}^{\prime}$ in the field varying from $0.0081 K_{0}^{\prime}$ to $81 K_{0}^{\prime}$.

As Fig. 2 shows, each converged solution yielded a different apparent "transition" location that was located further downstream with increasing initial dissipation rate values. At suffi- 
ciently high levels $\left(\varepsilon_{0}^{\prime}=81 K_{0}^{\prime}\right)$, the flow remained laminar throughout. Initially, this might seem like consistent behavior: an increased dissipation should reduce the level of turbulent kinetic energy $K$ and therefore should shift the position of "transition" further downstream. However, this rationale is flawed for two reasons; one more serious than the other from a CFD practitioner's point of view.

First, in the presence of mean shear (e.g. $S=\partial U / \partial y$ ), a laminar solution is characterised by very large values of the time-scale ratio, i.e., $S K / \varepsilon \gg 1$. Physically this implies that the turbulent time scale is much greater than the mean flow time scale and as such turbulence does not persist. But inspection of the "laminar" regions in the current solution shows that $S K / \varepsilon$ is in fact $\ll 1$. In other words, the $K-\varepsilon$ model is not really predicting laminar flow at all, but rather a pseudo-laminar behavior that will be shown in section III to be a stable fixed point of the equations.

Second, and even more serious is that the final converged solution should not depend on the initial condition in a steady-state computation at all! The converged solution should depend on the boundary conditions only, and in this case the boundary conditions were the same in all computations. It should be stressed that the solutions presented in Fig. 2 were very well converged solutions. The $L_{2}$-norm of the density residual dropped by more than 6 orders of magnitude, to approximately $1 \times 10^{-14}$, as shown in Fig. 3. The solutions after 25,000 multigrid cycles showed no perceptible differences from those solutions obtained after 2500 cycles.

\section{B. RAE 2822 Airfoil}

As a second example of anomalous behavior, the flow over an RAE 2822 airfoil at freestream Mach number $M_{\infty}=0.75$, angle-of-attack $\alpha=2.72$, and $R e=6.2 \times 10^{6}$ was computed. A plot showing the airfoil shape and resulting pressure contours for these conditions is given in Fig. 4 . There is a strong shock wave present on the airfoil upper surface near $65 \%$ chord, whereas the flow on the lower surface remains subsonic. In this example, the initial and boundary conditions were kept fixed but the numerical solution method was changed. The initial conditions and farfield boundary conditions in this case were set to $K_{\infty}^{\prime}=2.5 \times 10^{-8}$ and $\varepsilon_{\infty}^{\prime}=3.75 \times 10^{-8}$. This corresponded to a very low freestream turbulent intensity of $0.013 \%$ and a freestream eddy viscosity of $\mu_{t} / \mu_{\infty}=0.009$. These are typical values used in CFL3D. ${ }^{7}$

Figure 5 shows the skin-friction distribution on the lower surface of the airfoil obtained using two different numerical solution strategies for obtaining converged solutions. The converged results were completely different, with each suggesting a "transition" location in a different 
place. The first case was run using multigrid and 3 levels of mesh sequencing, with 2500 iterations on the coarse grid, followed by 2500 iterations on the medium grid, and finally 3000 iterations on the finest grid $(257 \times 97)$. The second case was run with multigrid and 2 levels of mesh sequencing, with 5000 iterations on the medium grid followed by 3000 iterations on the finest grid. Although not shown, both cases converged very well, with the $L_{2}$-norm of density residual reduced more than 3 orders of magnitude.

Both these examples show that caution needs to be exercised when using the $K-\varepsilon$ model. A numerically converged solution does not necessarily constitute the intended solution to the set of governing equations; it may depend on numerical parameters such as initial conditions and solution procedure. It is also important to mention here that many CFD practitioners have noticed that the $K-\varepsilon$ equations often fail to go fully turbulent, although the cause has never been identified before. In fact, it is customary to build in ad-hoc fixes to attempt to ensure that turbulence always develops. Some of these fixes include: (1) restarting $K-\varepsilon$ solutions from another turbulent solution, (2) setting initial conditions to have turbulent-like levels rather than freestream levels, and (3) imposing a temporary source term in the boundary layer. All of these fixes, in general, are workable ways to avoid the problem; but they do not shed any light on the reasons behind the problem and were not developed based on any firm rational foundation. As a consequence, their generality cannot be assured. In the following section an analysis is conducted that makes the reasons clear.

\section{Dynamical Systems Analysis}

A dynamical systems analysis can be used to determine the temporal dynamics associated with the numerical solution of systems of equations. ${ }^{10}$ A so-called nullcline analysis will also be used to identify some parametric restrictions on the $K-\varepsilon$ equations Eq. (1), in order to avoid arbitrary pseudo-laminar converged solutions.

It is possible to gain critical insight into the solution behavior for inhomogeneous turbulent flows through an analysis of the homogeneous form of Eq. (1). In its homogeneous limit, Eq. (1) can be written as a nonlinear, autonomous equation system in the form

$$
\begin{aligned}
\frac{d K^{*}}{d t^{*}} & =C_{\mu} \frac{K^{* 2}}{\varepsilon}-\varepsilon \\
\frac{d \varepsilon}{d t^{*}} & =C_{\varepsilon 1} C_{\mu} K^{*}-f_{2} C_{\varepsilon 2} \frac{\varepsilon^{2}}{K^{*}}
\end{aligned}
$$


where $K^{*}=S K\left(S \equiv \partial u / \partial y\right.$ is fixed in the analysis), and $t^{*}=S t$. There can be either one or two critical points in this system: $(i)$ the null vector with elements $K^{*}=\varepsilon=0$, and (ii), if it exists, is the intersection of the set of points with $d K^{*} / d t^{*}=0$ that lie on the $K^{*}$-nullcline described by

$$
\varepsilon= \pm \sqrt{C_{\mu}} K^{*}
$$

and the set of points with $d \varepsilon / d t^{*}=0$ that lie on the $\varepsilon$-nullcline described by

$$
\varepsilon= \pm \sqrt{\frac{C_{\mu} C_{\varepsilon 1}}{f_{2} C_{\varepsilon 2}}} K^{*}
$$

Realizability considerations dictate that only the positive roots need to be considered. At the intersection point of Eq. (7a) and Eq. (7b), $f_{2}=C_{\varepsilon 1} / C_{\varepsilon 2}$. Thus, this second critical point exists only if $f_{2}$ can achieve a value $C_{\varepsilon 1} / C_{\varepsilon 2}$ (which for the current set of closure coefficients is 0.7869). From Eq. (4) or Eq. (5), this means that the second critical point (ii) can exist only if $c_{1} \geq 1-C_{\varepsilon 1} / C_{\varepsilon 2}$ or $c_{3} \geq 1-C_{\varepsilon 1} / C_{\varepsilon 2}$ for these particular choices of $f_{2}$.

If stable, the critical points represent the possible steady-state solutions to the system, Eq. (6). Note that neither of these critical points is the so-called "turbulent" solution. In the analysis of homogenous turbulent flow, the "turbulent" solution grows without bound $\left(K^{*} \rightarrow \infty\right)$. The reason the analysis yields an unbounded growth in this case is that the mean flow field is fixed and unaffected by the turbulence, and this provides an infinite source of energy for the turbulence. In practical computations, this behavior is not seen because there is a two-way coupling between the turbulent and mean flow fields. The coupling allows for a steady-state to be reached. Diffusion, to be introduced later in the analysis, also plays an important role in practical computations.

The stability properties of the two critical points can be examined by linearizing about each critical point. The coefficient matrix of this linear system is the Jacobian matrix

$$
J=\left[\begin{array}{cc}
2 C_{\mu}\left(\frac{K^{*}}{\varepsilon}\right) & -C_{\mu}\left(\frac{K^{*}}{\varepsilon}\right)^{2}-1 \\
C_{\mu} C_{\varepsilon 1}+f_{2} C_{\varepsilon 2}\left(\frac{\varepsilon}{K^{*}}\right)^{2}-C_{\varepsilon 2}\left(\frac{\varepsilon^{2}}{K^{*}}\right) \frac{\partial f_{2}}{\partial K^{*}} & -2 f_{2} C_{\varepsilon 2}\left(\frac{\varepsilon}{K^{*}}\right)-C_{\varepsilon 2}\left(\frac{\varepsilon^{2}}{K^{*}}\right) \frac{\partial f_{2}}{\partial \varepsilon}
\end{array}\right] .
$$

To determine the nature of the critical points (e.g., if they are stable or unstable), the eigenvalues of this matrix are found at the critical points. Table 1 lists the possible types. A center indicates that trajectories orbit around the critical point. A stable critical point means that, when solving the equations, the particular point can be reached; an unstable critical point or a saddle point 
means that a numerical scheme will not converge to it (a saddle point is not stable in practice because orbits approach the critical point along one eigenvector, but then recede along the eigenvector associated with the unstable solution).

Table 1. Type of critical point as determined by eigenvalues of $J$.

\begin{tabular}{|c|c|}
\hline type of eigenvalues & critical point type \\
\hline \hline complex with zero real part & center \\
\hline complex with negative real part & stable focus \\
\hline complex with positive real part & unstable focus \\
\hline real and both negative & stable node \\
\hline real and both positive & unstable node \\
\hline real with one positive, one negative & saddle point \\
\hline \hline
\end{tabular}

It can be shown that, when it exists, the second critical point (ii) of the system Eq. (6) is always a saddle point. When Eq. (4) is used for $f_{2}$, the eigenvectors at the saddle point are:

$$
\left[\begin{array}{l}
\mathbf{X}_{1} \\
\mathbf{X}_{2}
\end{array}\right]=\left[\begin{array}{c}
2 \\
\sqrt{C_{\mu}}\left(1+C_{\varepsilon 1}\right)+C_{\varepsilon 2} \sqrt{C_{\mu}} D \ln (B) \pm \sqrt{A}
\end{array}\right]
$$

where $A=C_{\mu}\left(1-C_{\varepsilon 1}\right)^{2}+C_{\mu} C_{\varepsilon 2} D^{2} \ln ^{2}(B)+8 C_{\mu} C_{\varepsilon 2} D\left(C_{\varepsilon 1}-3\right) \ln (B), B=\left(C_{\varepsilon 2}-\right.$ $\left.C_{\varepsilon 1}\right) /\left(c_{1} C_{\varepsilon 2}\right)$, and $D=1-C_{\varepsilon 1} / C_{\varepsilon 2}$. When Eq. (5) is used for $f_{2}$, the eigenvectors at the saddle point are:

$$
\left[\begin{array}{l}
\mathbf{X}_{\mathbf{1}} \\
\mathbf{X}_{\mathbf{2}}
\end{array}\right]=\left[\begin{array}{c}
2 \\
\sqrt{C_{\mu}}\left(1+C_{\varepsilon 1}\right) \pm \sqrt{C_{\mu}\left(1-C_{\varepsilon 1}\right)^{2}-C_{\mu} C_{\varepsilon 2} D \ln (E)}
\end{array}\right]
$$

where $E=\left(C_{\varepsilon 2}-C_{\varepsilon 1}\right) /\left(c_{3} C_{\varepsilon 2}\right)$. The effect of the saddle point on the solution trajectories will be shown below when phase plots are drawn. But the more interesting critical point in this analysis is the first $(i)$ degenerate one $\left(K^{*}=\varepsilon=0\right)$, which corresponds to a pseudo-laminar solution. When Eq. (4) or Eq. (5) is used for $f_{2}$, and if the following condition is true,

$$
\frac{K^{*}}{\varepsilon} \ll 1 \quad \text { as } K^{*}=\varepsilon=0 \text { is approached, }
$$

then this degenerate point is either a center or a stable point. This stability of the degenerate 
critical point turns out to be the cause of the apparently arbitrary solution behavior demonstrated in section II.

In section C, the conditions for which Eq. (11) occur will be explored in greater detail. It will also be shown that stability of the degenerate point requires the second critical (saddle) point to exist. For now, however, the assertion is made that in practice Eq. (11) is quite often true for the system of equations given by Eq. (1) or Eq. (6). As a result, $R e_{T} \ll 1$ and $R e_{K} \ll 1$ near the critical point as well. Therefore, for $c_{1} \neq 1$ Eq. (4) is approximately $f_{2}=1-c_{1}$, and for $c_{1}=1$ it is approximately $f_{2}=c_{2} K^{* 4} /\left(S^{4} \nu^{2} \varepsilon^{2}\right)$. Similarly, for $c_{3} \neq 1$ Eq. (5) is approximately $f_{2}=1-c_{3}$, and for $c_{3}=1$ it is approximately $f_{2}=c_{4} K^{* 1 / 2} d /\left(\nu S^{1 / 2}\right)$. Using these expressions, along with expressions for the derivatives of Eq. (4) and Eq. (5) with respect to $K^{*}$ and $\varepsilon$, the eigenvalues of the matrix in Eq. (8) near the degenerate critical point can be determined. These are given in Table 2 for the two $f_{2}$ expressions and various combinations of their coefficients. In all cases but one, the degenerate critical point is stable. (The "center" type of critical point is also not desirable in this context, because solutions can become locked in an orbit and fail to converge. However, no known models actually use $c_{1}=1$ in Eq. (4), so from now on the analysis focuses only on the more common cases where the critical point is stable.)

Table 2. Eigenvalues near the $K^{*}=\varepsilon=0$ critical point, with $K^{*} / \varepsilon \ll 1$. The computations in section II employed a model that corresponds to the third alternative.

\begin{tabular}{c|c|c}
\hline$f_{2}$ function $\left(c_{2}, c_{4}>0\right)$ & type of eigenvalues & critical point type \\
\hline \hline $1-c_{1} \exp \left(-c_{2} R e_{T}^{2}\right)$, & complex, near-zero real part & center \\
$c_{1}=1$ & real, both negative for $c_{1}<\left(C_{\varepsilon 2}-1\right) / C_{\varepsilon 2}$ & stable node \\
\hline $1-c_{1} \exp \left(-c_{2} R e_{T}^{2}\right)$, & stable focus \\
$0<c_{1}<1$ & complex, negative real part for $c_{1}>\left(C_{\varepsilon 2}-1\right) / C_{\varepsilon 2}$ & stable focus \\
\hline $1-c_{3} \exp \left(-c_{4} R e_{K}\right)$, & real, both negative for $c_{3}<\left(C_{\varepsilon 2}-1\right) / C_{\varepsilon 2}$ & \\
$c_{3}=1$ & stable node \\
\hline $1-c_{3} \exp \left(-c_{4} R e_{K}\right)$, & complex, negative real part for $c_{3}>\left(C_{\varepsilon 2}-1\right) / C_{\varepsilon 2}$ & stable focus \\
$0<c_{3}<1$ & & \\
\hline \hline
\end{tabular}

Equations (7a) and (7b) can be sketched for a case when the two nullclines intersect. Inspection of Eq. (6a) and Eq. (6b) then shows the following to apply:

$$
\frac{d K^{*}}{d t^{*}}=0 \text {, on Eq. (7a) } \frac{d K^{*}}{d t^{*}}<0 \text {, left of Eq. (7a) } \frac{d K^{*}}{d t^{*}}>0 \text {, right of Eq. (7a) (12a) }
$$




$$
\frac{d \varepsilon}{d t^{*}}=0 \text {, on Eq. (7b) } \frac{d \varepsilon}{d t^{*}}<0, \text { above Eq. (7b) } \frac{d \varepsilon}{d t^{*}}>0 \text {, below Eq. (7b). }
$$

Combining this information with the knowledge of the behavior at the critical points, a clear mapping of the phase-plane trajectories can be drawn, as shown in Fig. 6. The eigenvectors $\left(\mathbf{X}_{1}, \mathbf{X}_{2}\right)$ at the saddle point are shown along with dividing curves (dashed lines) that separate regions of different trajectory behavior. Clearly, any initial condition above dividing curve 1 will eventually end up at the degenerate critical point.

An actual phase-plane portrait can be constructed by computing the right hand sides of Eq. (6) for a large number of $K^{*}$ and $\varepsilon$ values. These computed values then correspond to $\partial K^{*} / \partial t^{*}$ and $\partial \varepsilon / \partial t^{*}$ at each particular point in nondimensional $K^{*}-\varepsilon$ phase space, and the trajectory (how $K^{*}$ and $\varepsilon$ change with time or with iteration) can be computed as well.

An example is shown in Fig. 7, using Eq. (5) for $f_{2}$ with $c_{3}=1, c_{4}=2 / 25, S^{\prime}=1569$, $d^{\prime}=2.945 \times 10^{-4}$ (typical results using Eq. (4) yield a similar behavior). The nullclines are shown along with the phase space trajectories. It is clear that this figure matches the sketch in Fig. 6 in character: $K^{*}=\varepsilon=0$ is a stable attractor, and the other critical point (near $K^{*}=0.19, \varepsilon=0.057$ in this particular case) is a saddle point. The true turbulent solution is obtained when $K^{*}$ grows (exponentially); in other words, the expected turbulent solution only occurs when the $K^{*}$ and $\varepsilon$ values follow the trajectories in the lower right or far upper right

parts of the plot. This figure demonstrates that there are many regions in the map for which the solution converges toward the degenerate critical point $K^{*}=\varepsilon=0$.

It is also interesting to look at the phase space trajectories of the case with $f_{2}=1$, shown in Fig. 8. Here, the two nullclines do not cross, so there is no saddle point (i.e., no second critical point). The trajectories now behave like the ones to the right of the saddle point in Fig. 7; regardless of the initial condition, the solution always goes to the turbulent solution and never to the degenerate one.

\section{A. Accounting for Diffusion}

Inhomogeneous effects that necessarily occur in any practical calculation can be represented by the viscous diffusion term and the gradient diffusion models for the turbulent transport, represented by $\nu_{t} / \sigma_{K}$ and $\nu_{t} / \sigma_{\varepsilon}$. Since the consideration here is for high Reynolds number flows, viscous diffusion effects can be neglected in the turbulent region, and it is only necessary to adequately represent the effects of the turbulent tranport in both the kinetic energy and energy dissipation rate equations. It suffices for the purposes here to simply assume that the transport 
effects act over a distance $l$ given by $K^{3 / 2} / \varepsilon$. Thus, qualitative estimates for the transport terms can be written as

$$
\begin{gathered}
\frac{\partial}{\partial x_{j}}\left(\frac{\nu_{t}}{\sigma_{K}} \frac{\partial K}{\partial x_{j}}\right) \sim \mathcal{O}\left(\frac{K^{3}}{\varepsilon l^{2}}\right) \sim \mathcal{O}(\varepsilon)=C_{\mu K}^{*} \varepsilon \\
\frac{\partial}{\partial x_{j}}\left(\frac{\nu_{t}}{\sigma_{\varepsilon}} \frac{\partial \varepsilon}{\partial x_{j}}\right) \sim \mathcal{O}\left(\frac{K^{2}}{l^{2}}\right) \sim \mathcal{O}\left(\frac{\varepsilon^{2}}{K}\right)=C_{\mu \varepsilon}^{*} \frac{\varepsilon^{2}}{K},
\end{gathered}
$$

with $C_{\mu K}^{*}$ and $C_{\mu \varepsilon}^{*}$ as unknown coefficients. Using these estimates for the transport terms allows them to be grouped with the destruction terms. Thus, an equation set that accounts for inhomogeneity can be written as

$$
\begin{aligned}
\frac{d K^{*}}{d t^{*}} & =C_{\mu} \frac{K^{* 2}}{\varepsilon}-\left(1-C_{\mu K}^{*}\right) \varepsilon \\
\frac{d \varepsilon}{d t^{*}} & =C_{\varepsilon 1} C_{\mu} K^{*}-\left(f_{2} C_{\varepsilon 2}-C_{\mu \varepsilon}^{*}\right) \frac{\varepsilon^{2}}{K^{*}} .
\end{aligned}
$$

It is clear to see that the effect of the additional diffusion-type terms is merely to tilt the nullclines up, making them steeper. As will be shown in section B, this effect is needed to achieve agreement between theory and computation. The relative shapes and positions of the phase space trajectories are a function of the relative magnitudes of the (a priori unknown) $C_{\mu K}^{*}$ and $C_{\mu \varepsilon}^{*}$. In this case, the nullclines intersect to form a saddle point when $f_{2}=$ $\left[C_{\varepsilon 1}\left(1-C_{\mu K}^{*}\right)+C_{\mu \varepsilon}^{*}\right] / C_{\varepsilon 2}$.

\section{B. Comparison of Computations with Analysis}

Through the foregoing analysis, it is clear that the equations themselves (when they contain an $f_{2}$ function in the $\varepsilon$-equation destruction term) can cause degenerate solutions to occur. But how well does the theory compare with actual RANS computations? Although the actual RANS computations are much more complicated than the analytical model because the mean flow $S$ and the actual diffusion terms vary in time and space, we can look at these varying values at specific points from the earlier flat plate computation, for example, and choose representative levels over the latter part of the temporal development. These representative levels can then be inserted into Eq. (14) when computing the theoretical phase-space trajectories for comparison.

Figures 9 and 10 show the results using this procedure at two different points in the flow field. The first figure shows computed results at a point in the boundary layer that eventually converged to a pseudo-laminar degenerate result, along with the theoretical trajectories. Here, 
diffusion effects were negligible, and there is excellent agreement between theory and computation. The second figure is for a point further downstream that became fully turbulent. Here, the effect of diffusion was important and needed to be taken into account in the theory; values used for $C_{\mu K}^{*}$ and $C_{\mu \varepsilon}^{*}$ were 0.28 and 0.59 , respectively, based on representative levels seen in the flat plate computation. There is again excellent agreement between theory and computation.

\section{Avoiding Arbitrary Steady-State Solutions}

Earlier, Eq. (11) was given as a condition for which the degenerate critical point was either a center or a stable point. The interrelationship between this condition and the value of $f_{2}$ is now explored. Consider the equation for $K^{*} / \varepsilon$ :

$$
\frac{d\left(K^{*} / \varepsilon\right)}{d t^{*}}=-\frac{\mathcal{P}}{\varepsilon}\left(C_{\varepsilon 1}-1\right)-\left(1-C_{\varepsilon 2} f_{2}\right)
$$

where $\mathcal{P} / \varepsilon=C_{\mu} K^{* 2} / \varepsilon^{2}$. From this equation it is immediately apparent that for $d\left(K^{*} / \varepsilon\right) / d t^{*}<$ 0 (which must be true for $K^{*} / \varepsilon$ to be driven toward zero), the following must hold:

$$
f_{2}<\frac{\mathcal{P}}{\varepsilon} \frac{C_{\varepsilon 1}-1}{C_{\varepsilon 2}}+\frac{1}{C_{\varepsilon 2}} .
$$

If this inequality is not satisfied, then $K^{*} / \varepsilon$ remains finite, and the eigenvalue analysis of the Jacobian matrix Eq. (8) shows that the $K^{*}=\varepsilon=0$ critical point is unstable, and no longer an attractor for the degenerate solution. This suggests a method to avoid the problem of arbitrary solutions for steady-state computations: compute $f_{2}$ as usual with Eq. (4) or Eq. (5), but then limit it via

$$
f_{2}^{\prime}=\min \left[1, \max \left(f_{2}, \frac{\mathcal{P}}{\varepsilon} \frac{C_{\varepsilon 1}-1}{C_{\varepsilon 2}}+\frac{1}{C_{\varepsilon 2}}\right)\right],
$$

during the early transient stages of a steady-state computation. This limiter does not allow the value of $f_{2}$ to go below the critical level defined by Eq. (16). Once turbulence has been established, then the limiter can be removed. This analysis does not lead to a method for avoiding arbitrary solutions in time-dependent computations. Such computations are prone to anomalous behavior as well.

Another important observation is that the right hand side in Eq. (16) is less than $C_{\varepsilon 1} / C_{\varepsilon 2}$ for $\mathcal{P} / \varepsilon<1$. Because the second critical (saddle) point exists only if $f_{2}$ can achieve a value $C_{\varepsilon 1} / C_{\varepsilon 2}$, this implies that a second critical point is necessary in order for the degenerate point ( $K^{*}=\varepsilon=0$ ) to be stable, when using the current equation set. 


\section{Extension to More General Form}

To summarize, the analysis suggests that the presence of two critical points for a commonlyused form of the $K-\varepsilon$ equations has the potential to produce anomalous pseudo-laminar behavior. The degenerate point associated with the pseudo-laminar solution $\left(K^{*} / \varepsilon \ll 1 ; K^{*}=\varepsilon=0\right)$ is stable only if there exists a second critical point given by $f_{2}=C_{\varepsilon 1} / C_{\varepsilon 2}$.

One can write the homogeneous limit of the $K-\varepsilon$ equations in more general form:

$$
\begin{aligned}
\frac{d K^{*}}{d t^{*}} & =C_{\mu}^{*} \frac{K^{* 2}}{\varepsilon}-\varepsilon \\
\frac{d \varepsilon}{d t^{*}} & =\frac{\varepsilon}{K^{*}}\left(C_{\varepsilon 1}^{*} C_{\mu}^{*} \frac{K^{* 2}}{\varepsilon}-C_{\varepsilon 2}^{*} \varepsilon\right),
\end{aligned}
$$

where the new variables $C_{\mu}^{*}, C_{\varepsilon 1}^{*}$, and $C_{\varepsilon 2}^{*}$ can now each include functions of the solution (for example, low-Reynolds-number models ${ }^{9}$ often use a function $f_{\mu}$ that is a part of $C_{\mu}^{*}$ ). In the more general form Eq. (18), the second critical point is now defined by

$$
C_{\varepsilon 2}^{*}=C_{\varepsilon 1}^{*} .
$$

The analysis strongly suggests that any $K-\varepsilon$ model for which $C_{\varepsilon 2}^{*} \leq C_{\varepsilon 1}^{*}$ somewhere in the flow has the potential to yield an arbitrary pseudo-laminar solution.

\section{Concluding Remarks}

A peculiar problem inherent in a widely-used form of the $K-\varepsilon$ turbulence model has been demonstrated and analyzed. This problem has a potentially large impact on practical CFD computations. Use of an $f_{2}$ function multiplying the destruction term of the dissipation rate equation was shown to cause portions of the flow field to converge to a degenerate pseudo-laminar condition. Most disturbingly, this condition is highly dependent on numerical parameters such as initial conditions and solution procedure. In other words, RANS solutions using this particular form of the $K-\varepsilon$ model can easily yield arbitrary fully-converged solutions with pseudo-laminar regions of varying size. Time-dependent computations are also susceptible to the anomalous behavior.

A nullcline analysis was used to analyze the homogenous form of the equations, followed by a form that approximately accounts for the effect of diffusion. The analysis clearly demonstrated the reasons for the anomalous behavior of this turbulence model: the degenerate solution was 
a stable fixed point under certain conditions, causing the numerical method to converge there. The analysis also led to a methodology for preventing the anomalous behavior in steady-state solutions using the current equation set.

The results presented here also suggested that any $K-\varepsilon$ model for which the coefficient multiplying the destruction term in the $\varepsilon$ equation can be less than or equal to the coefficient multiplying the production term has the potential to produce arbitrary pseudo-laminar solutions.

\section{References}

${ }^{1}$ Pope, S. B., Turbulent Flows, Cambridge University Press, Cambridge, 2000.

${ }^{2}$ Durbin, P. and Pettersson-Reif, B. A., Statistical Theory and Modeling of Turbulent Flows, John Wiley and Sons, New York, 2001.

${ }^{3}$ Mohammadi, B. and Pironneau, O., Analysis of the K-Epsilon Turbulence Model, John Wiley \& Sons Ltd., Chichester, 1994.

${ }^{4}$ Speziale, C. G. and Mhuiris, N. M. G., "On the prediction of equilibrium states in homogeneous turbulence," J. Fluid Mech., Vol. 209, 1989, pp. 591-615.

${ }^{5}$ Speziale, C. G., Gatski, T. B., and Mhuiris, N. M. G., "A critical comparison of turbulence models for homogeneous shear flows in a rotating frame," Phys. Fluids A, Vol. 2, 1990, pp. 1678-1684.

${ }^{6}$ Speziale, C. G., Sarkar, S., and Gatski, T. B., "Modelling the pressure-strain correlation of turbulence: an invariant dynamical systems approach,” J. Fluid Mech., Vol. 227, 1991, pp. 245-272.

${ }^{7}$ Krist, S. L., Biedron, R. T., and Rumsey, C. L., “CFL3D User's Manual (Version 5.0),” Nasa tm-1998208444, June 1998.

${ }^{8}$ Morrison, J. H., "Flux Difference Split Scheme for Turbulent Transport Equations,” Aiaa paper 90-5251, 1990.

${ }^{9}$ Patel, V. C., Rodi, W., and Scheuerer, G., "Turbulence models for near-wall and low Reynolds number flows: a review," AIAA J., Vol. 23, No. 9, 1985, pp. 1308-1319.

${ }^{10}$ Gerstner, W. and Kistler, W. M., Spiking Neuron Models, Single Neurons, Populations, Plasticity, Cambridge University Press, Cambridge, 2002. 


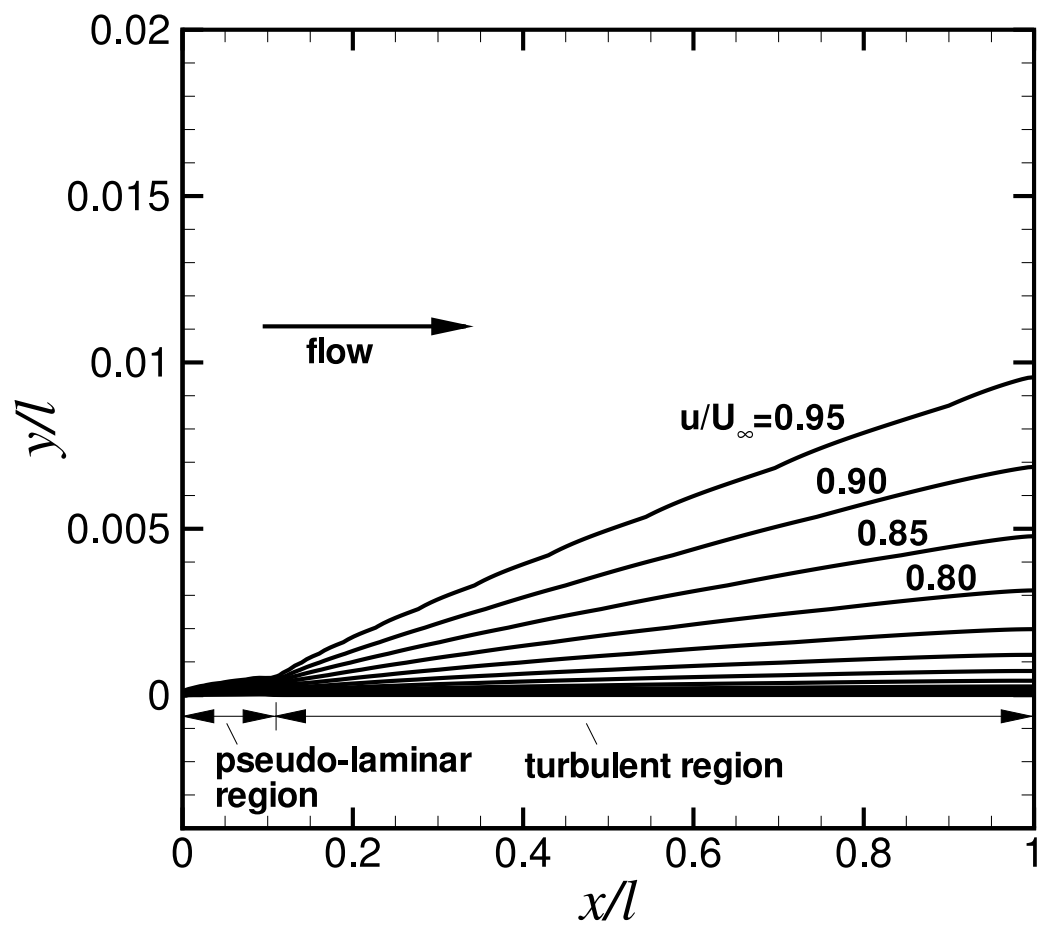

Figure 1. Example flat plate solution showing $u / U_{\infty}$ contours for initial condition $\varepsilon_{0}^{\prime}=8.1 K_{0}^{\prime} ; M_{\infty}=0.2$, $R e=6.0 \times 10^{6}(l=$ length of plate). 


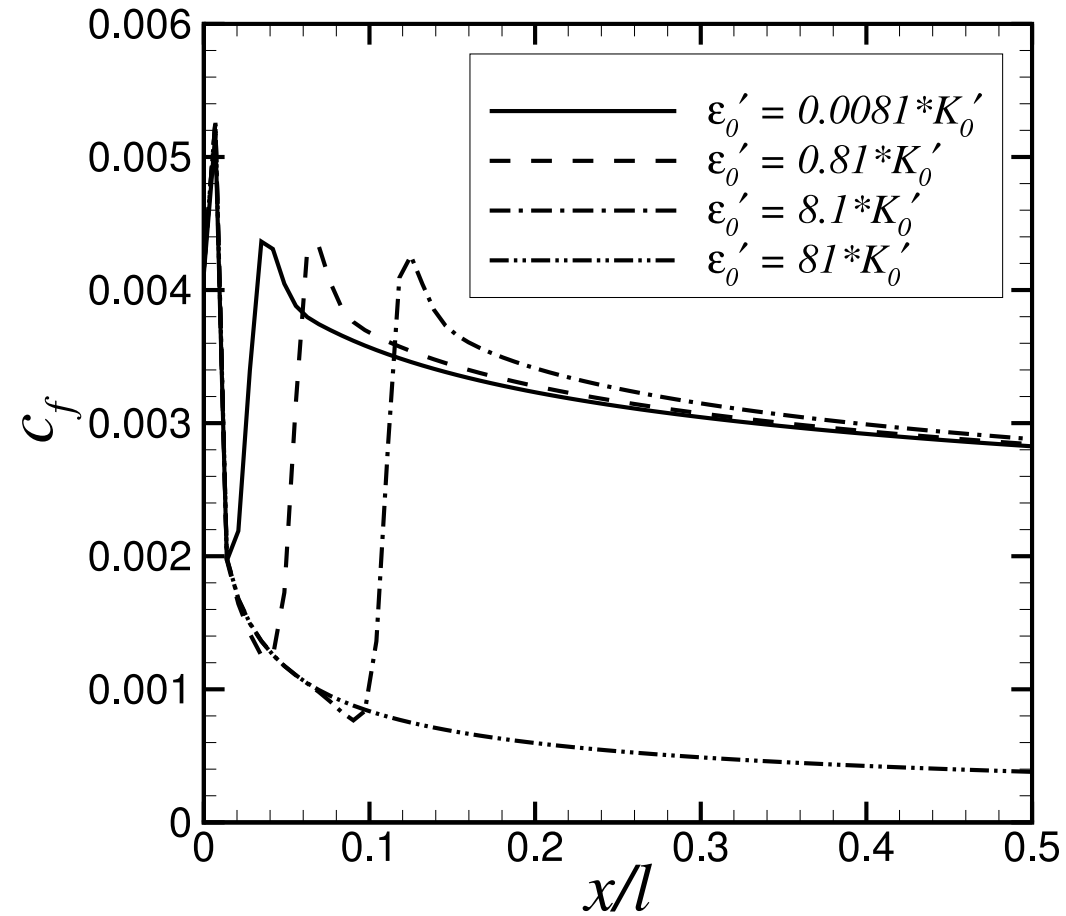

Figure 2. Streamwise variation of skin-friction coefficient on front half of flat plate as a function of initial conditions $\left(K_{0}^{\prime}=6.0 \times 10^{-6}\right)$. 


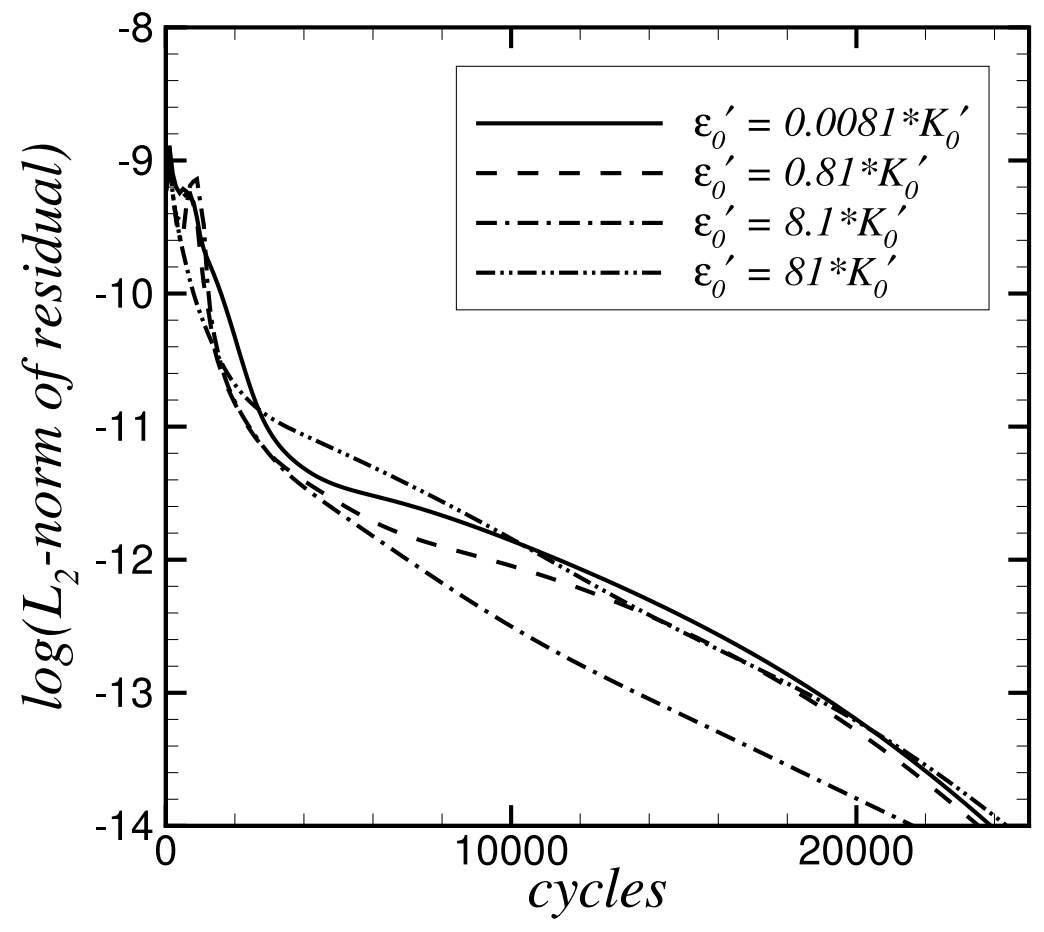

Figure 3. Convergence history for flat plate computations. 


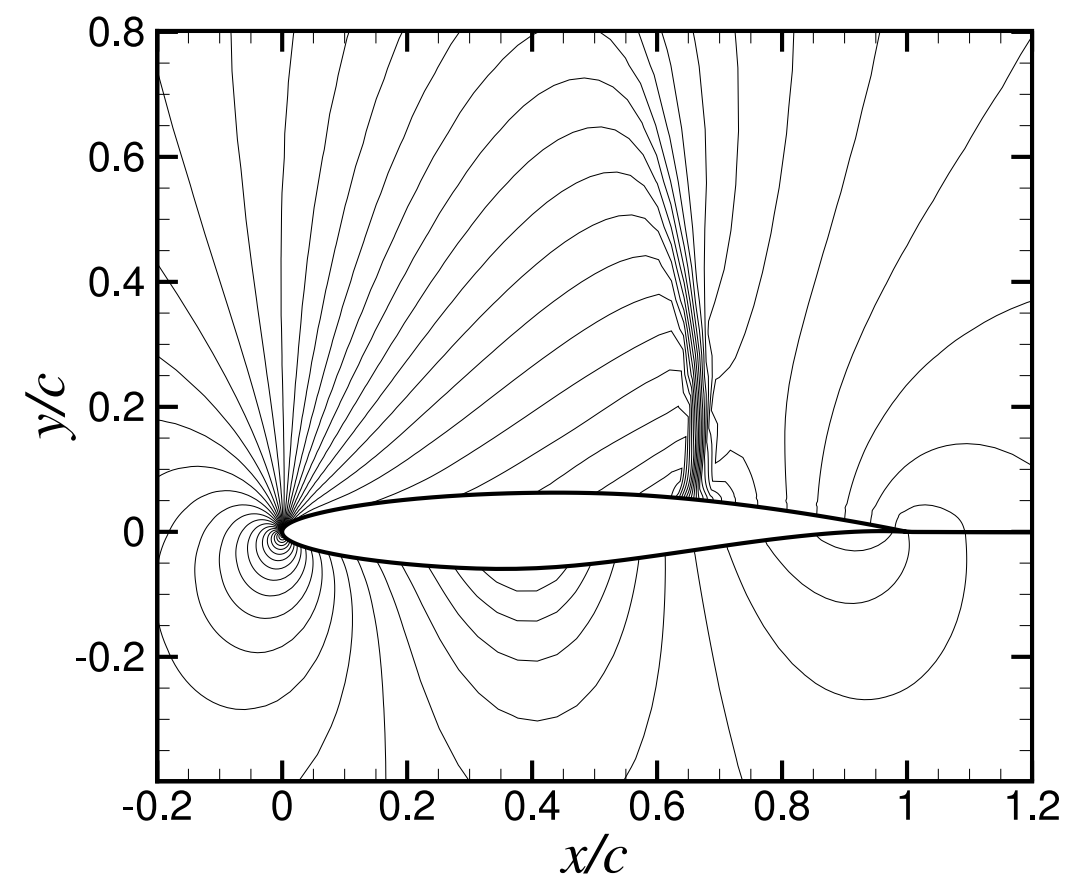

Figure 4. Example RAE 2822 airfoil solution showing static pressure contours; $M_{\infty}=0.75, \alpha=2.72$, $R e=6.2 \times 10^{6}(c=$ chord length). 


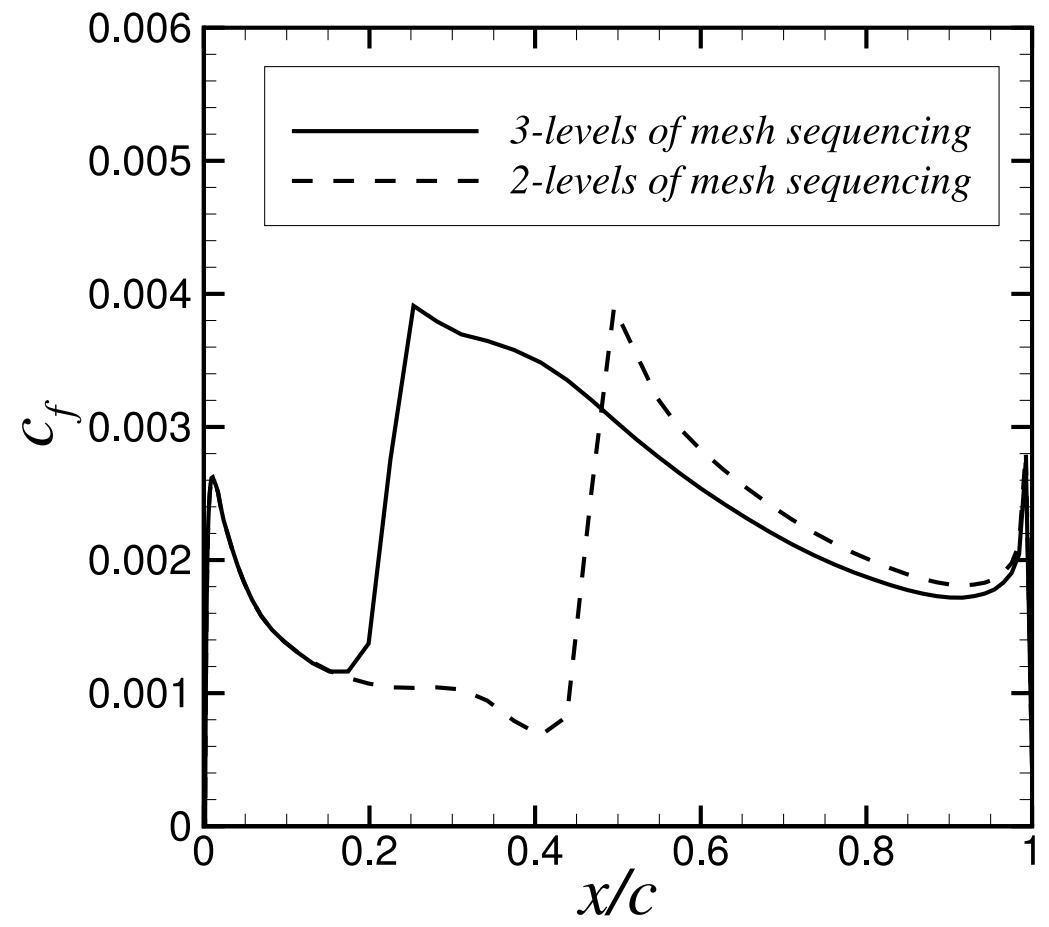

Figure 5. Streamwise variation of skin-friction coefficient on RAE 2822 airfoil lower surface for two different solution procedures (both procedures converged). 


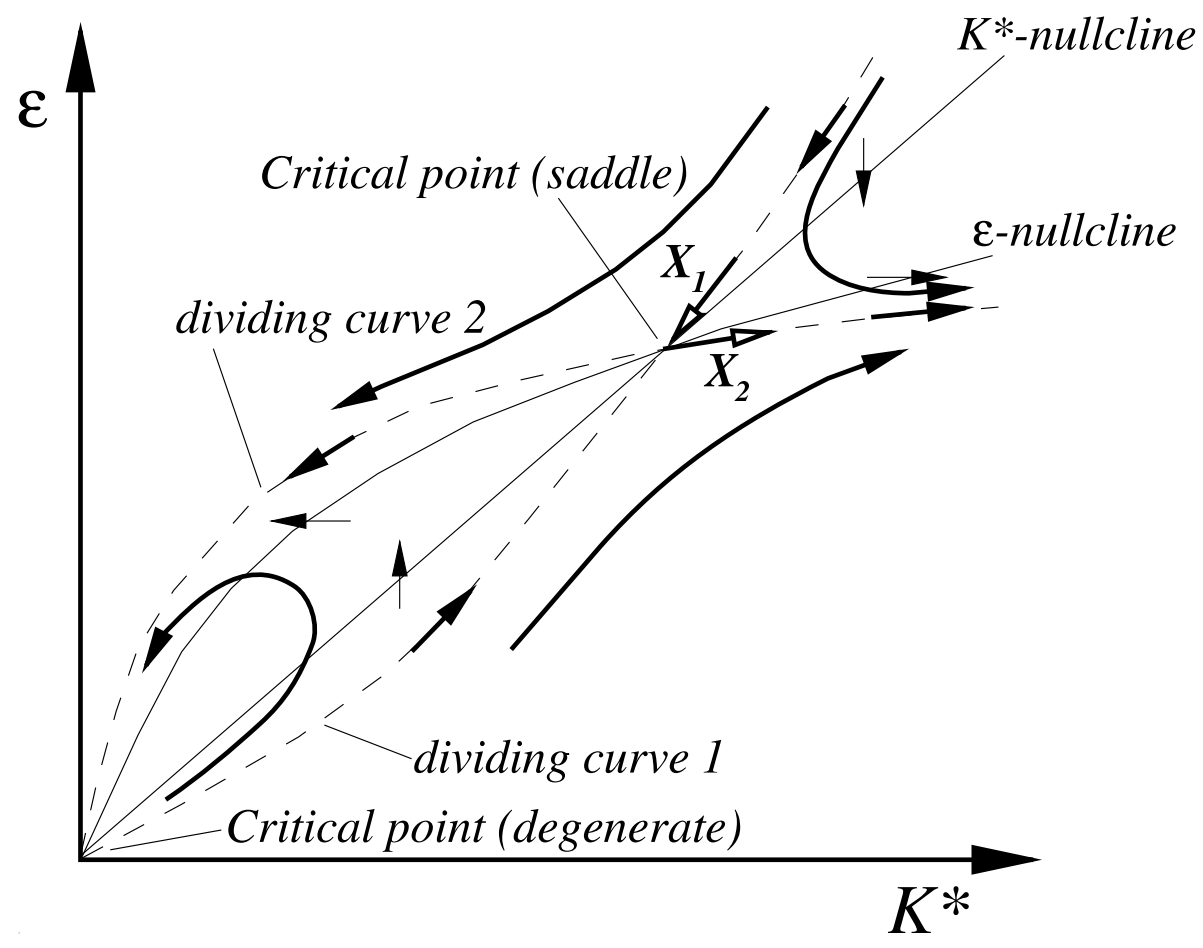

Figure 6. Sketch of nullclines and critical points from Eq. (7a) and Eq. (7b) and resulting $K^{*}-\varepsilon$ phase diagram $\left(K^{*}=S K\right)$; dividing curves (dashed lines) divide trajectories near critical points. 


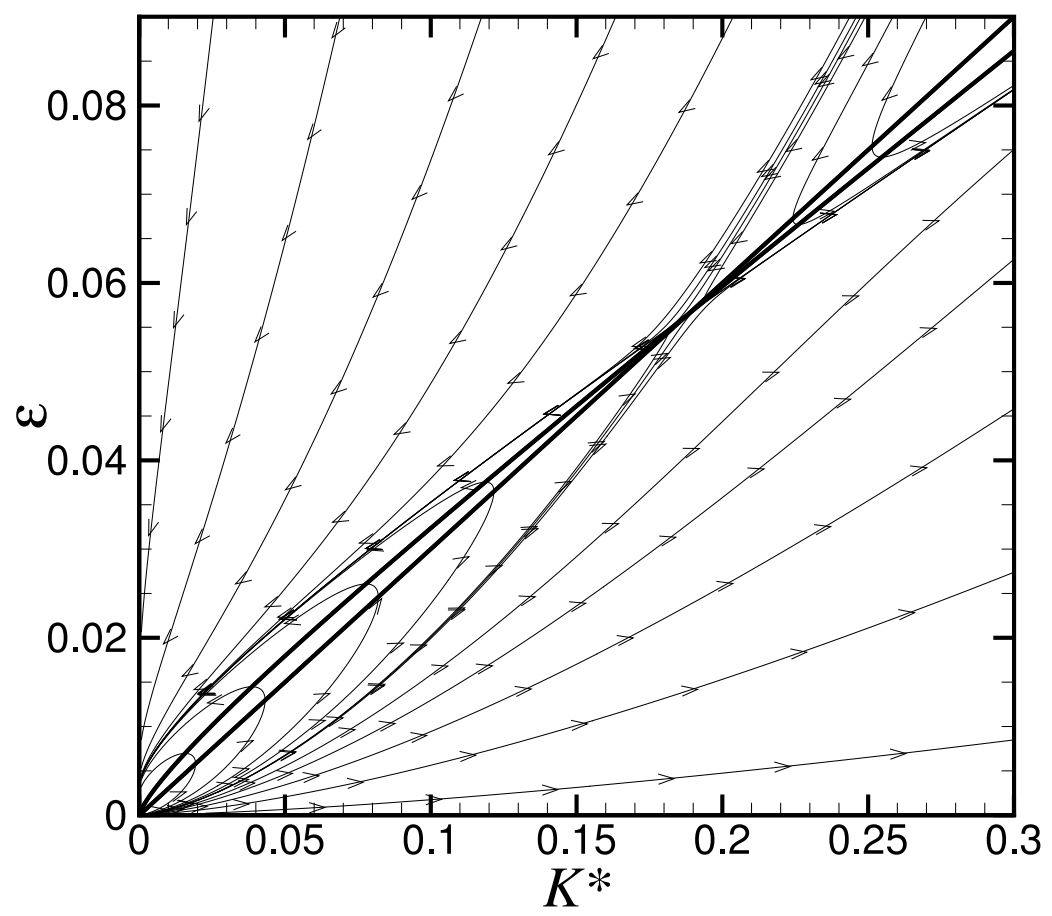

Figure 7. Example phase-plane portrait of Eq. (6) showing nullclines (two oblique lines going from lower left to upper right) and trajectories (lines with arrows) with $f_{2}$ given by Eq. (5). 


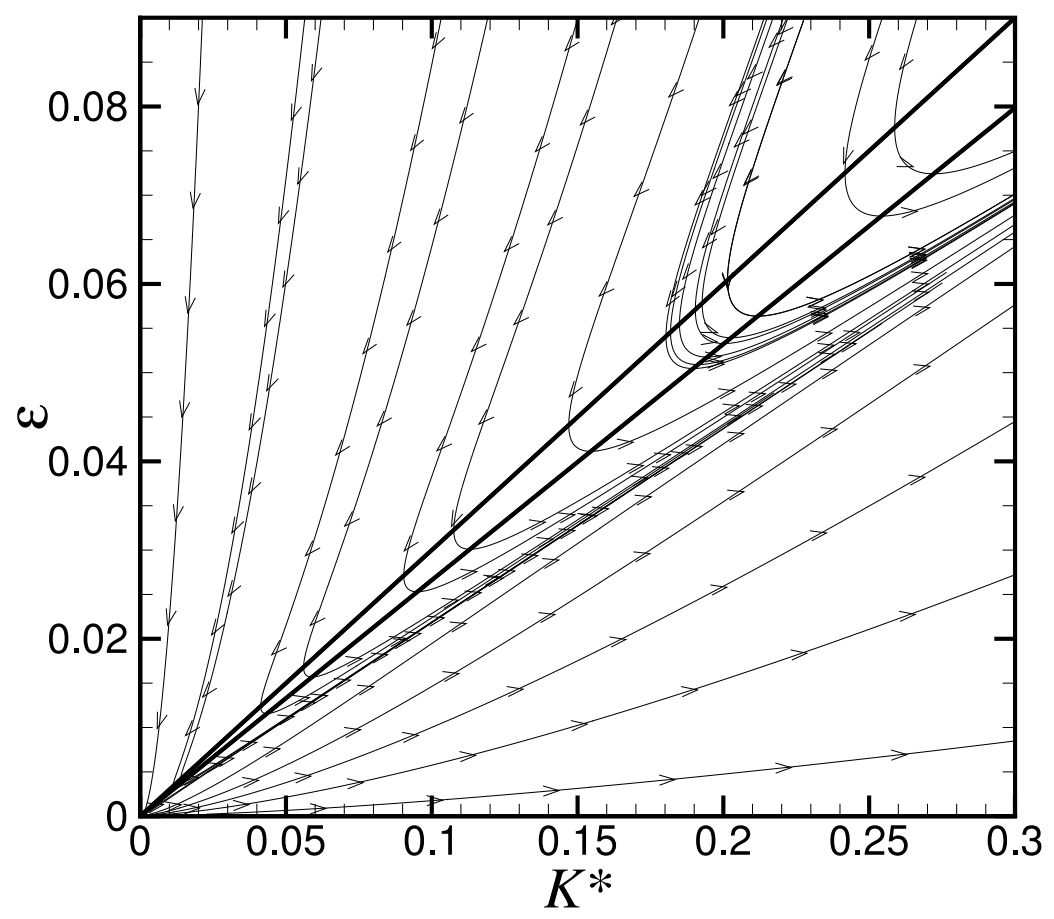

Figure 8. Example phase-plane portrait of Eq. (6) showing nullclines (two oblique lines going from lower left to upper right) and trajectories (lines with arrows) with $f_{2}=1$. 


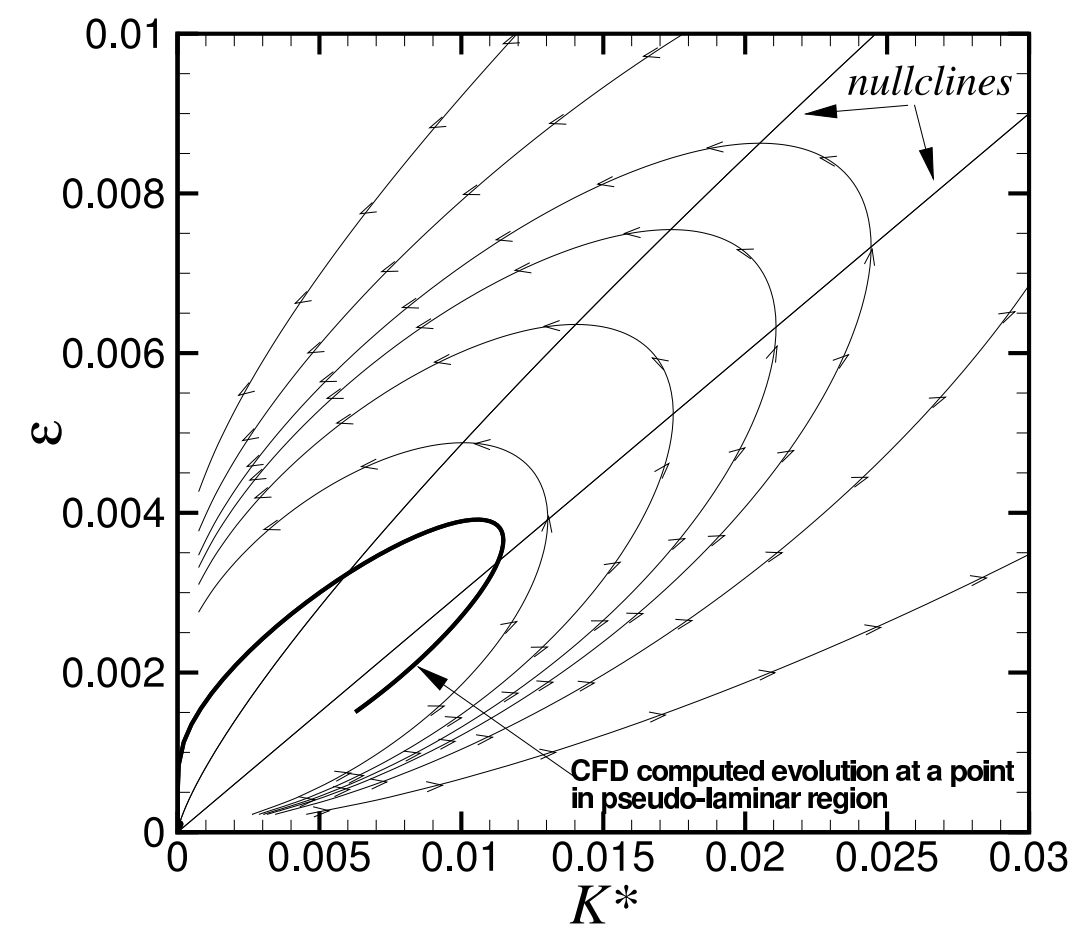

Figure 9. Comparison of theory with computed result at a point in pseudo-laminar region of the flat plate computation. 


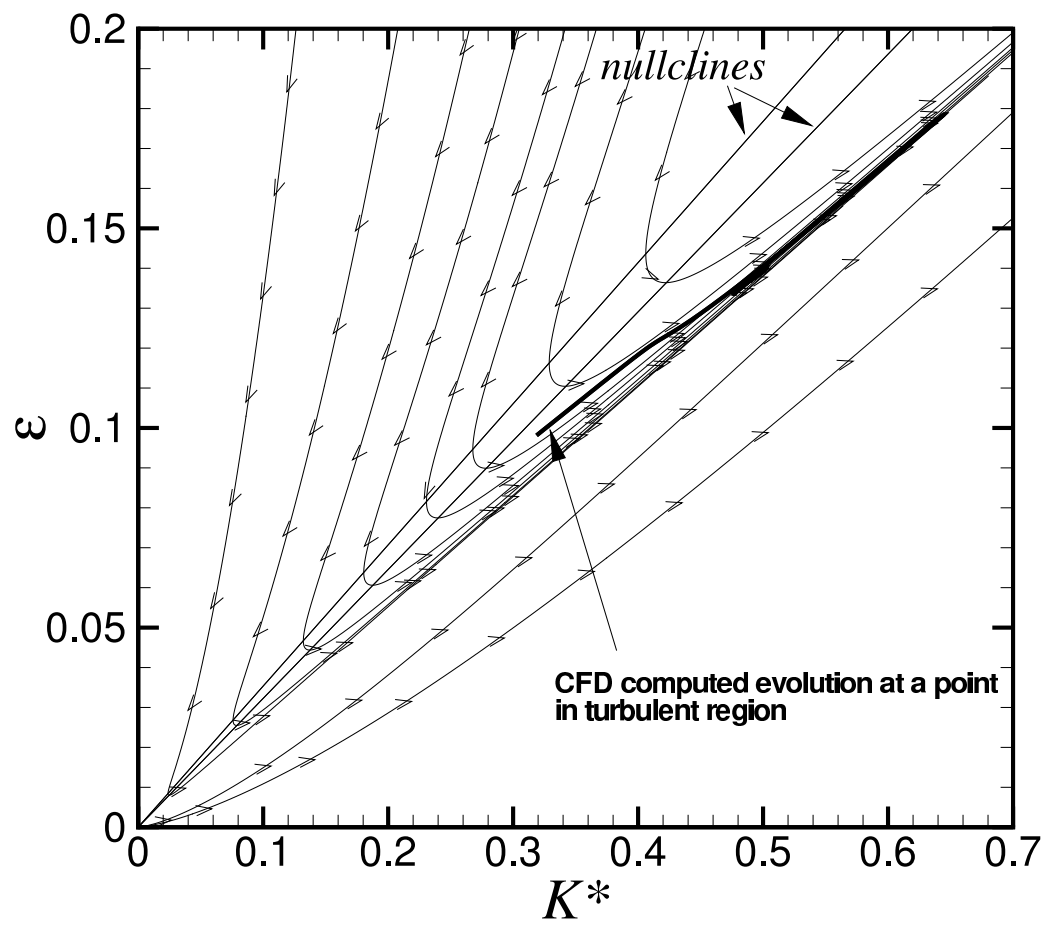

Figure 10. Comparison of theory with computed result at a point in turbulent region of the flat plate computation. 